\title{
Shadow Removal in Outdoor Video Sequences by Automatic Thresholding of Division Images
}

\author{
Srinivasa Rao Dammavalam, Claudio Piciarelli, Christian Micheloni, \\ and Gian Luca Foresti \\ University of Udine, Italy
}

\begin{abstract}
Several video-based applications, such as video surveillance, traffic monitoring, video annotation, etc., rely on the correct detection and tracking of moving objects within the observed scene. Even though several works have been proposed in the field of moving object detection, many of them do not consider the problem of segmenting real objects from their shadows. The shadow is considered part of the object, thus leading to possibly large errors in the subsequent steps of object localisation and tracking. In this paper we propose a shadow detection algorithm able to remove shadows from the blobs of moving objects, using division images and Expectation-Maximization histogram analysis. Experimental results prove that the use of the proposed method can significantly increase the performance of a video analysis system.
\end{abstract}

\section{Introduction}

Moving shadow detection and removal is a fundamental and critical task for accurate object detection in many computer vision applications, including visual traffic monitoring, automated remote video surveillance and people tracking. Shadow regions are usually adjacent to object points and in many commonly used segmentation techniques shadows and objects are merged in a single blob, causing errors in object tracking and classification.

The problem has generally been addressed by using geometric or photometric properties of shadows. Prati et al. 1] give a comprehensive literature survey of the available methods that deal with moving shadow identification. In 1 the requirements of moving shadow detection methods are detailed and quantitative and qualitative comparison between different known approaches are given. Bevilacqua 2 proposed a work on the detection of moving shadows in the context of an outdoor traffic scene for video surveillance applications. The proposed algorithm relies on some photometric properties concerning shadows; the method is mainly based on multi-gradient operations applied on the division image to discover the shadow regions. In [3] shadow detection for moving objects is done using a three-stage shadow identification scheme without imposing restrictions on the number of light sources, illumination conditions, surface orientations and object sizes. In the first step, background segmentation is done using a mixture of Gaussians technique; then, pixel-based decisions are made by comparing the 
current frame with the background model to differ shadows from the actual foreground. Finally, the result is improved using blob-level reasoning which works on geometric constraints of identified shadow and foreground blobs. Cucchiara et al. 4 present a novel technique based on the HSV colour space for shadow detection and removal used in a system for moving object detection and tracking. In the Sakbot system [5] the technique has been exploited at two levels, both for improving segmentation and background update. In [6] Weiss proposed a technique to derive intrinsic, shadow-free images based on the concept of decomposing an image into its reflectance and illumination components; however it requires multiple images of a static scene under different lighting conditions and it is ineffective in removing vague shadows. In [7] a method is proposed in which invariant images are obtained by finding an image that is orthogonal to the direction of shadow intensity and colour change. In 8] Support Vector Machines (SVM) are used with a Gaussian kernel in order to find complex shadow boundaries; a co-training algorithm is used to improve the classification accuracy even when a small number of training examples is available.

As it can be seen, many relevant works on shadow detection and removal rely on radiometric properties of shadows, which can be used to extract invariant properties for the shadowed regions; however, these works often require the manual definition of fixed thresholds, thus limiting the general applicability of the method. In this paper we will propose a totally automated method for shadow detection and removal for outdoor scenes. The method uses division images in order to extract proper shadow features and performs ExpectationMaximization-based histogram analysis to automatically compute the required thresholds.

\section{System Overview}

The proposed shadow detection technique is developed in order to enhance the performance of a video surveillance system whose general architecture is shown in Figure 1. Images are acquired by a static colour camera and successively processed in order to identify zones of high intensity/chromatic variations (change detection). Even though the final aim of the change detection step is to identify the presence of moving objects in the scene, the shadows of moving objects are detected as well, thus causing erroneous data to be processed by the high-level modules; this justifies the introduction of a shadow detection and removal step.

Whenever a new image $I(x, y)$ is acquired, it is used to update a background model, this is an image of the scene without moving objects. We use a Gaussian model, in which each background pixel is represented by a running-average mean $\mu(x, y)$ and a variance $\sigma^{2}(x, y)$ computed using the previously acquired frames. Change detection is achieved by comparing the current frame with the background model according to the formula

$$
M(x, y)= \begin{cases}1 & \text { if }|I(x, y)-\mu(x, y)|>\sigma(x, y) \\ 0 & \text { otherwise }\end{cases}
$$




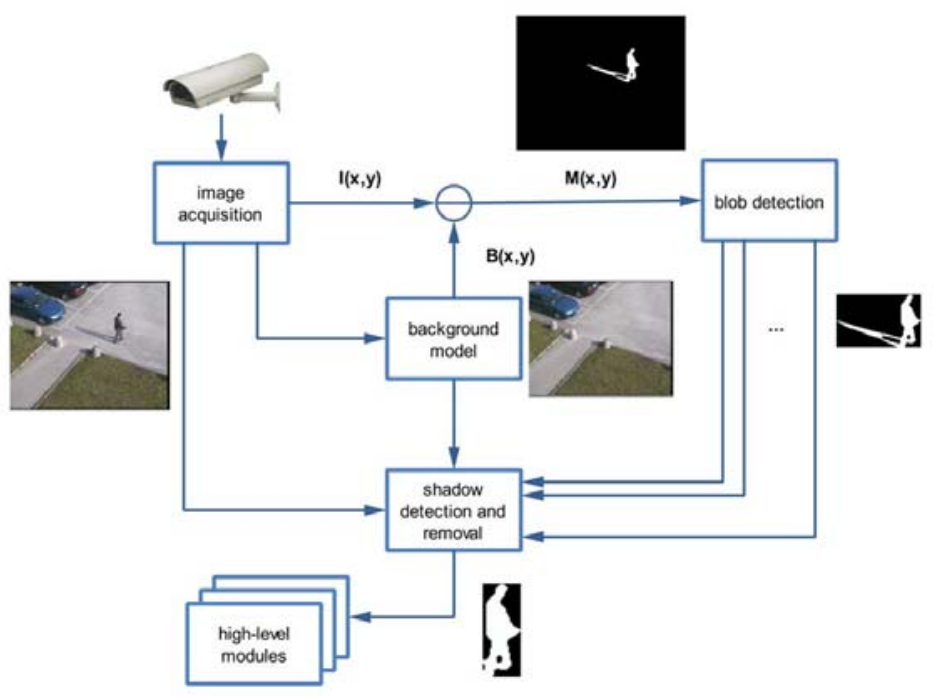

Fig. 1. System architecture

where $M(x, y)$ is the binary mask of foreground moving regions. Image $M$ is processed in order to detect connected components and their bounding boxes, representing the moving objects within the scene. Each bounding box is processed by the shadow removal module in order to detect and remove the shadows included in the foreground mask. Once shadows have been removed, the highlevel modules of the system can process the obtained data in order to perform visual surveillance tasks. These modules typically involve the classification and tracking of the moving objects, as well as a behaviour analysis step in order to detect potentially suspicious, dangerous or forbidden events 9 .

\section{Shadow Detection}

As stated before, shadow detection and removal is a essential step in order to acquire good-quality data for the high-level processing modules. Many of the shadow detection algorithms described in section 11 take into account a priori information, such as the geometry of the scene and of the moving objects. In this method, we do not deal with geometric constraints, however we will assume that the sequences are acquired in outdoor environments under direct sunlight.

The proposed method is based on simple radiometry considerations about how light is spread across the scene when an object is lit by a light source [5]. We rely on the Lambertian model, in which the brightness of a lighted surface depends on the light source and object properties, but not on the position of the 
observer. The radiance (the amount of light reaching the camera) $L$ at a given pixel $(x, y)$ is thus defined as

$$
L(x, y)=E(x, y) \rho(x, y)
$$

where $E(x, y)$ is the irradiance, the amount of light coming from the light source and hitting the object at the point of coordinates $(x, y)$ in the image plane, while $\rho(x, y)$ is the surface reflectance, an intrinsic property of the object about how much light it can reflect. While $\rho$ depends exclusively on the lighted object, the irradiance depends both on the light source and its position respect to the surface normals. Moreover, we note that in real-world scenarios, irradiance depends on at least two light components, one coming directly from the main light source (e.g. the sun), and the other being the ambient light, a diffuse light coming from the surrounding environment (e.g. the sky) 4]. If no ambient light is considered, shadows would be totally black, which is generally not the case. We will call the light source intensity and the ambient light intensity respectively $e_{l}$ and $e_{a}$. The irradiance can thus be defined as

$$
E(x, y)= \begin{cases}e_{a}+e_{l} N(x, y) \cdot L & \text { for lit objects } \\ e_{a} & \text { for shadows }\end{cases}
$$

where $N(x, y)$ and $L$ respectively are unit vectors representing the surface normal and the light direction. The dot product shows how the irradiance depends on the angle between light rays and the surface normals; note that under the assumption of outdoor scenes with the sun as a primary light source, $L$ can be considered constant.

Let now consider a frame $I(x, y)$, its foreground mask $M(x, y)$ and its corresponding background image $B(x, y)$. We introduce the notion of division image, a real-valued image defined as

$$
D(x, y)=B(x, y) / I(x, y)
$$

For each point $(x, y)$, three cases can arise:

1. the point does not belong to the foreground mask, in this case $D(x, y) \approx 1$ (approximation is due to image noise);

2. the point is included in the foreground mask, and belongs to a shadow. In this case, by using eqs. 1 and 2 , equation 3 becomes

$$
D(x, y)=\frac{L_{I}(x, y)}{L_{B}(x, y)}=\frac{E_{I}(x, y)}{E_{B}(x, y)}=\frac{e_{a}+e_{l} N(x, y) \cdot L}{e_{a}}>1
$$

if we consider the case of a locally flat ground plane where the shadow is projected, then $N(x, y)$, and consequently $D$, are constant across all the shadow region;

3. the point is included in the foreground mask and belongs to a real moving object; in this case no hypotheses can be made on $D(x, y)$ since it will depend on the intensity/colour properties of the object itself. 


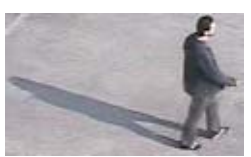

(a)

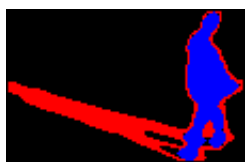

(b)

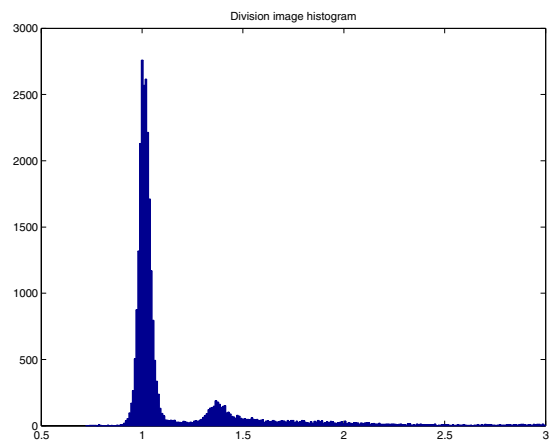

(c)

Fig. 2. Histogram of a division image

We thus hypothesize that the division image histogram will have two major peaks, one centred around 1 and denoting standard background pixels, one centred on a value greater than 1 and denoting shadows, as shown in Figure 2 No assumptions can be made on moving object pixels: they could produce a third peak in the histogram if both the background and the foreground objects have uniform colours, or more probably they will be scattered across the histogram without any particular distribution.

Even though some papers have already used this assumption [5], they relied on the detection of the shadow peak by manually defining two fixed upper and lower thresholds. This method requires manual tuning and it is not generic, since different scenes subject to different lighting conditions can require different thresholds. We here propose to automatically detect the peaks by using the Expectation-Maximization (EM) algorithm 10. EM is a statistical, iterative method for finding maximum likelihood estimates of the parameters of a probabilistic model depending on latent variables. It is based on the iteration of two main steps: expectation, in which the hidden data are estimated based on the current parameters, and maximization, in which the parameters are estimated by likelihood maximization using the current hidden and known data. The process is iterated until convergence. In our case, the method is applied to a mixtureof-Gaussians model, in which the hidden data are the mean and variance of two Gaussians that must fit the division image histogram. By using EM, the two peaks can thus be detected; we also tested the case of 3 Gaussians (in order to model a possible peak due to the real moving object) but without noticeable performance improvement. Once the Gaussians are detected, the one with higher mean is associated to shadow pixels. Any pixel such that $|D(x, y)-\mu|<\sigma$ is then classified as a shadow pixel and removed from the foreground mask $M$; the resulting image is then enhanced by using morphological operators. The whole shadow detection procedure is depicted in Figure 3. Finally, note that the above procedure is defined for single-channel images, but the extension to the colour case is straightforward: the process can be applied separately to the three 


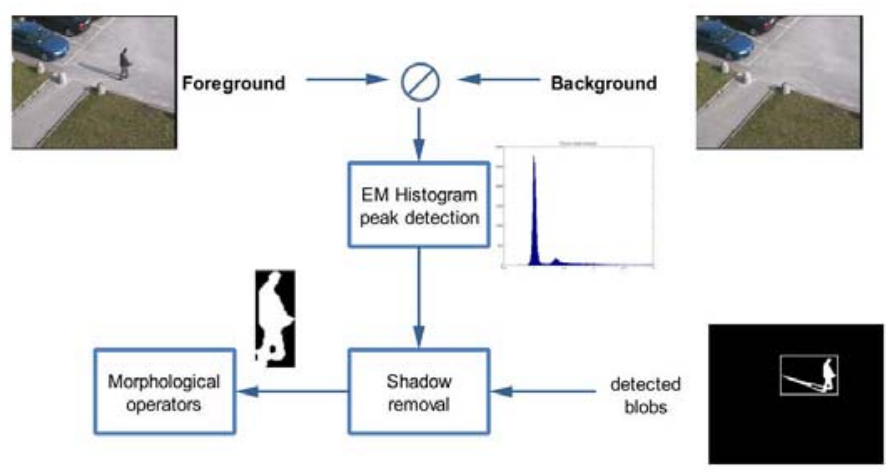

Fig. 3. The shadow detection process

red, green and blue channels, the pixel is finally classified as shadow only if all the three tests are positive (all the experiments proposed in sec. 4 have been performed on colour images).

\section{Experimental Results}

In order to evaluate the performance of the proposed shadow detection and removal system, we acquired several outdoor sequences during a sunny day, in order to have sharp shadows. All the sequences are composed of colour images acquired by a static camera at the resolution of $360 \times 290$ pixel, and involve a moving person within a passageway or in a parking lot. All the images are initially smoothed with a Gaussian kernel, and moving objects are detected using the technique described in section 2 Then, for each detected bounding box, shadow detection is applied in order to remove shadow pixels. Figure 4 allows a visual evaluation of the obtained results: some frames from the acquired sequences are shown together with the classification mask. In the mask, each nonblack pixel was initially classified as foreground by the change detection module. The foreground pixels were then further classified as shadows (red pixels) or real moving objects (blue pixels). As it can be seen, the shadow detection technique managed to remove the shadows while keeping a large part of the original real moving object.

The main aim of our technique is to improve the performance of the subsequent high-level processing modules, in particular the tracking one. Since our tracking system is based on the shape and position of the bounding box rectangle for each moving object, it is a natural choice to use bounding boxes in order to give numerical measurements of the error introduced by our method. For each frame considered for performance evaluation, a bounding box enclosing the moving object is thus manually defined and used as ground truth. The ground truth is then compared both with the bounding box detected by the change detection module and the new bounding box after shadow removal: we want to prove that 


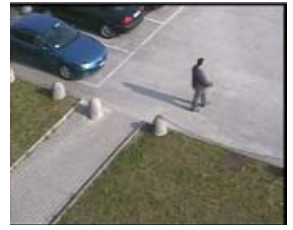

(a)

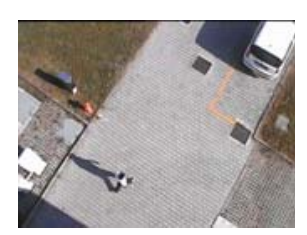

(e)

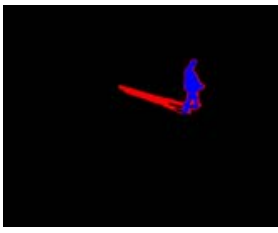

(b)

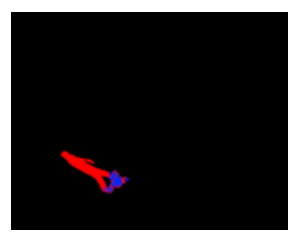

(f)

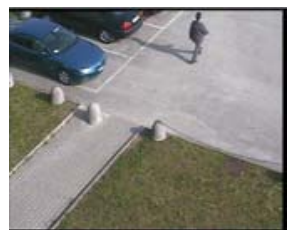

(c)

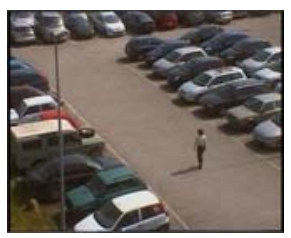

(g)

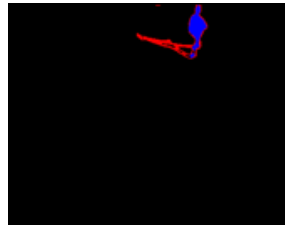

(d)

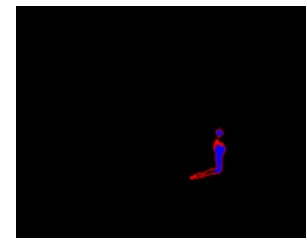

(h)

Fig. 4. Some example images. (a) (c), (e), (g) original images; (b), (d) (f), (h) the corresponding processed images, red areas are shadows, blue areas are foreground moving objects.

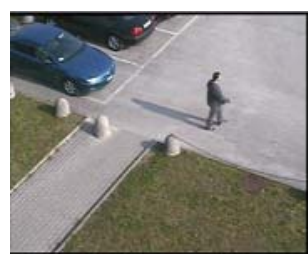

(a)

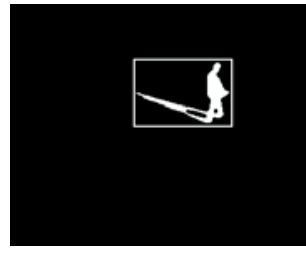

(b)

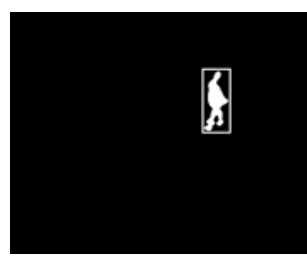

(c)

Fig. 5. Bounding boxes for a given moving object. (a) input frame; (b) detected bounding box without shadow removal; (c) detected bounding box with shadow removal.

the use of the shadow removal module always leads to better results if compared with the basic approach. An example of the data used in the evaluation is shown in Figure 5. where both the automatically computed bounding boxes are shown.

In order to numerically measure the difference between two bounding boxes, we used the same metric (the Dice coefficient) proposed in the ETISEO contest [11. If two bounding boxes $\mathrm{A}$ and $\mathrm{B}$ are given, the Dice coefficient $D$ is defined as

$$
D=\frac{2|A \cap B|}{|A|+|B|}
$$

where $|\cdot|$ is the area of a given bounding box. The results are given in table 1 the sequences used are the ones shown in figure 4 (sequence 1: figure (a) \& (c), sequence 2: figure (e), sequence 3: figure (g) 1 . As it can be seen the

${ }^{1}$ Sequences available at

http://avires.dimi.uniud.it/papers/iciap09/sequences.zip 
Table 1. Comparing the computed bounding boxes with ground truth data, 1 means perfect bounding box matching. The first column shows the results obtained without shadow removal; the second column refers to shadow removal with fixed thresholds as in 4]; the last column refers to shadow removal with the proposed method.

\begin{tabular}{|l||c|c|c|}
\hline Sequence & no shadow removal & $\begin{array}{c}\text { shadow removal } \\
\text { (fixed thresholds) }\end{array}$ & $\begin{array}{c}\text { shadow removal } \\
\text { (proposed method) }\end{array}$ \\
\hline \hline seq 1, frame 160 & 0.3456 & 0.3529 & 0.7585 \\
seq 1, frame 264 & 0.3160 & 0.3577 & 0.7578 \\
seq 1, frame 570 & 0.4059 & 0.4153 & 0.7300 \\
seq 1, frame 1055 & 0.4051 & 0.4480 & 0.7173 \\
seq 1, frame 1156 & 0.3455 & 0.3829 & 0.8281 \\
seq 1, frame 1371 & 0.3405 & 0.4460 & 0.8387 \\
seq 1, frame 1478 & 0.3894 & 0.5124 & 0.8663 \\
seq 1, frame 1807 & 0.3994 & 0.4100 & 0.8203 \\
seq 1, frame 1878 & 0.3670 & 0.4348 & 0.8331 \\
seq 1, frame 1926 & 0.3431 & 0.3936 & 0.8104 \\
seq 2, frame 272 & 0.3858 & 0.4427 & 0.7669 \\
seq 2, frame 297 & 0.3797 & 0.6781 & 0.7087 \\
seq 2, frame 323 & 0.4494 & 0.5817 & 0.7778 \\
seq 2, frame 579 & 0.4056 & 0.4233 & 0.8476 \\
seq 2, frame 625 & 0.4006 & 0.5331 & 0.6180 \\
seq 2, frame 704 & 0.4419 & 0.3934 & 0.8595 \\
seq 2, frame 938 & 0.4159 & 0.4934 & 0.8942 \\
seq 2, frame 1000 & 0.3817 & 0.4768 & 0.7120 \\
seq 2, frame 1037 & 0.3794 & 0.4826 & 0.5845 \\
seq 2, frame 1072 & 0.3564 & 0.6619 & 0.6947 \\
seq 3, frame 329 & 0.3586 & 0.3840 & 0.3655 \\
seq 3, frame 367 & 0.4018 & 0.4146 & 0.4846 \\
seq 3, frame 556 & 0.3722 & 0.4706 & 0.5902 \\
seq 3, frame 590 & 0.3537 & 0.3838 & 0.7490 \\
seq 3, frame 764 & 0.3253 & 0.4437 & 0.5275 \\
seq 3, frame 919 & 0.4308 & 0.4976 & 0.8759 \\
seq 3, frame 1126 & 0.3030 & 0.4131 & 0.4545 \\
seq 3, frame 1156 & 0.2941 & 0.4042 & 0.4632 \\
seq 3, frame 1390 & 0.3856 & 0.3863 & 0.5042 \\
seq 3, frame 1669 & 0.5197 & 0.5029 & 0.5422 \\
\hline
\end{tabular}

introduction of the shadow removal step systematically leads to better results than the basic approach. Moreover, in table 1 we also give comparison with a fixed threshold approach, in which no EM histogram analysis is done in order to automatically detect the peaks in the histogram. In this experiment, we used the fixed thresholds proposed in 4]. Again it is evident that the automatic computation of the thresholds leads to better results than previously proposed methods. 


\section{Conclusions}

In this paper, a novel approach for the detection and removal of moving shadows in outdoor video sequences has been presented and discussed. The approach relies on the use of division images, whose histogram gives strong hints on the possible classification of each pixel in the scene. The histogram peaks are automatically detected by means of Expectation-Maximization and allow the automatic detection of shadow pixels in foreground masks. The experimental results clearly show that the introduction of the proposed shadow removal technique gives a large improvement on the performance of the system.

\section{Acknowledgements}

This work was partially supported by the Italian Ministry of University and Scientific Research within the framework of the project "Ambient Intelligence: event analysis, sensor reconfiguration and multimodal interfaces" (PRIN 20072008). Srinivasa Dammalavam thanks the Italian Ministry of University and Research for the financial support.

\section{References}

1. Prati, A., Mikic, I., Trivedi, M.M., Cucchiara, R.: Detecting moving shadows: Algorithms and evaluation. IEEE Trans. on Pattern Analysis and Machine Intelligence 25(7), 918-923 (2003)

2. Bevilacqua, A.: Effective shadow detection in traffic monitoring applications. Journal of WSCG 11(1), 57-64 (2003)

3. Joshi, A.J., Atev, S., Masoud, O., Papanikolopoulos, N.: Moving shadow detection with low- and mid-level reasoning. In: IEEE International Conference on Robotics and Automation, Rome, Italy, pp. 4827-4832 (2007)

4. Cucchiara, R., Grana, C., Piccardi, M., Prati, A., Sirotti, S.: Improving shadow suppression in moving object detection with hsv color information. In: IEEE Proc. Intelligent Transportation Systems, Oakland, CA, USA, pp. 334-339 (2001)

5. Cucchiara, R., Grana, C., Piccardi, M., Prati, A.: Statistic and knowledge-based moving object detection in traffic scenes. In: IEEE Proc. Intelligent Transportation Systems, Dearborn, MI, USA, pp. 27-32 (2000)

6. Weiss, Y.: Deriving intrinsic images from image sequences. In: IEEE International Conference on Computer Vision, Vancouver, BC, Canada, pp. 68-75 (2001)

7. Finlayson, G.D., Hordley, S.D., Drew, M.S.: On the removal of shadows from images. IEEE Trans. Pattern Analysis and Machine Intelligence 28(1), 59-68 (2006)

8. Joshi, A.J., Papanikolopoulos, N.P.: Learning to detect moving shadows in dynamic environments. IEEE Trans. Pattern Analysis and Machine Intelligence 30(11), 2055-2063 (2008)

9. Micheloni, C., Piciarelli, C., Foresti, G.: How a visual surveillance system hypothesizes how you behave. Behavior Research Methods 38(3), 447-455 (2006)

10. Moon, T.: The expectation-maximization algorithm. IEEE Signal Processing Magazine 13(6), 47-60 (1996)

11. Nghiem, A., Bremond, F., Thonnat, M., Valentin, V.: ETISEO, performance evaluation for video surveillance systems. In: Proc. of Advanced Video and Signal-based Surveillance, London, UK, pp. 476-481 (2007) 